Herzschr Elektrophys 2021 · 32:510-523 https://doi.org/10.1007/s00399-021-00796-x Angenommen: 1. Juli 2021

Online publiziert: 2. August 2021

(c) Springer Medizin Verlag GmbH, ein Teil von Springer Nature 2021

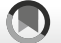

Check for
updates

Die Mitglieder der Bundesfachgruppe Herzschrittmacher und Defibrillatoren werden am Beitragsende gelistet.

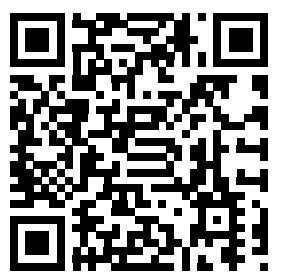

QR-Code scannen \& Beitrag online lesen

\section{Jahresbericht 2019 des Deutschen Herzschrittmacher- und Defibrillator-Registers - Teil 1: Herzschrittmacher}

\author{
Fachgruppe Herzschrittmacher und Defibrillatoren beim \\ IQTIG - Institut für Qualitätssicherung und Transparenz im \\ Gesundheitswesen
}

\author{
A. Markewitz ${ }^{1}$ - Bundesfachgruppe Herzschrittmacher und Defibrillatoren \\ 'Bendorf, Deutschland
}

\section{Inhaltsverzeichnis}

- Einleitung

- Datenbasis

- Datenvolumen

- Demografische Daten

- Implantationen

- EKG-Indikation zur Schrittmacherimplantation

- Auswahl der Schrittmachersysteme

- Operationsdaten

- Aggregatwechsel

- Revisionen/Systemwechsel/ Explantationen

- Kommentar mit internationalem Vergleich

- Datenbasis

- EKG-Indikationen zur Schrittmachertherapie

- Schrittmachersystemauswahl

- Operationsdaten

- Zusammenfassung und Ausblick

- Literatur

- Tabellenverzeichnis

- Abbildungsverzeichnis

\section{Einleitung}

Der 19. Jahresbericht des Deutschen Herzschrittmacher- und Defibrillator-Registers kann zum vierten Mal keine Ergebnisse berichten, die auf der sekundären Nutzung der bei den verpflichtenden Maßnahmen der Qualitätssicherung erhobenen Daten basieren. Das entsprechende Verfahren ist zwar wieder zugänglich, die inzwischen geforderten Details bei der Antragstellung sind aber ohne Unterstützung durch externe Expertise von den Autoren des Berichts nicht zu erbringen. Wir hoffen allerdings, dass diese Expertise demnächst zur Verfügung steht und dadurch im Bericht für das Jahr 2020 die Daten wieder etwas detaillierter betrachten werden können. Damit fehlen aber in diesem Bericht weiter viele der früher üblichen Auswertungen. Da zudem inzwischen viele Datenfelder dem Prinzip der Datensparsamkeit zum Opfer gefallen sind, ist dieser Bericht erneut deutlich kürzer als zuvor.

Des Weiteren ist dieser Bericht der letzte, der von der Fachgruppe Herzschrittmacher und Defibrillatoren publiziert werden kann. Aufgrund einer grundsätzlichen Änderung der Verfahren in der externen Qualitätssicherung durch Inkrafttreten der „Richtlinie zur datengestützten einrichtungsübergreifenden Qualitätssicherung (DeQS-RL)“ wird es in Zukunft keine „Fachgruppen“, sondern „Expertengremien auf Bundesebene" geben, die mit einem etwas geänderten Aufgabenspektrum die Arbeit der ehemaligen Fachgruppe als fachliches Beratungsgremium für das IQTIG fortsetzen werden. Diese Änderung sieht aber auch eine geänderte personelle Zusammensetzung sowie ein Bewerbungsverfahren vor, so dass zum Zeitpunkt der Erstellung dieses 
Tab. 1 Übersicht ausgewerteter Datensätze im Vergleich zu den Vorjahren, inkl. Minimaldatensätze

\begin{tabular}{|l|l|l|l|}
\hline Datenbasis & $\mathbf{2 0 1 7}$ & $\mathbf{2 0 1 8}$ & 2019 \\
\hline Krankenhäuser & 1102 & 1085 & 1073 \\
\hline 09/1: Implantationen & 957 & 916 & 907 \\
\hline 09/2: Aggregatwechsel & 923 & 891 & 879 \\
\hline 09/3: Revisionen/Systemwechsel/Explantationen & \multicolumn{3}{|l|}{} \\
\hline Eingriffe & 77.283 & 75.437 & 75.760 \\
\hline 09/1: Implantationen & 17.085 & 16.061 & 16.338 \\
\hline 09/2: Aggregatwechsel & 11.383 & 10.933 & 10.523 \\
\hline 09/3: Revisionen/Systemwechsel/Explantationen & 105.751 & 102.431 & 102.621 \\
\hline Alle 3 Leistungsbereiche (Summe) & & &
\end{tabular}

Tab. 2 Operationsvolumina (Implantationen, Aggregatwechsel und Revisionen) der meldenden Krankenhäuser

\begin{tabular}{|c|c|c|c|}
\hline \multirow{2}{*}{$\begin{array}{l}\text { Anzahl gemeldeter Herzschritt- } \\
\text { macheroperationen }\end{array}$} & \multicolumn{3}{|c|}{ Krankenhäuser } \\
\hline & 2017 & 2018 & 2019 \\
\hline \multicolumn{4}{|l|}{ Neuimplantationen ${ }^{a}$} \\
\hline$<20$ & 293 & 232 & 210 \\
\hline 20 und mehr & 806 & 772 & 770 \\
\hline \multicolumn{4}{|l|}{ Aggregatwechsel } \\
\hline$<20$ & 624 & 732 & 721 \\
\hline 20 und mehr & 333 & 206 & 201 \\
\hline \multicolumn{4}{|c|}{ Revisionen/Systemwechsel/Explantationen ${ }^{a}$} \\
\hline$<20$ & 774 & 737 & 725 \\
\hline 20 und mehr & 150 & 135 & 134 \\
\hline \multicolumn{4}{|c|}{ 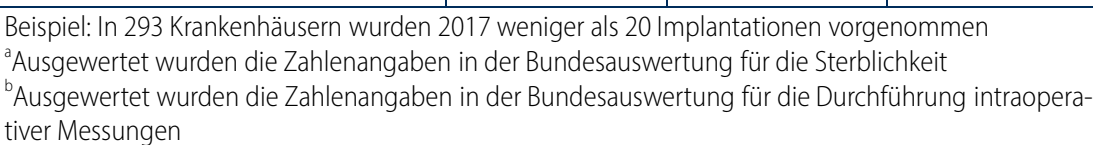 } \\
\hline
\end{tabular}

Tab. 3 Vollzähligkeit der ausgewerteten Datensätze bzw. Krankenhäuser im Jahr 2018

\begin{tabular}{|l|l|l|l|l|}
\hline Tab. 3 Vollzähligkeit der ausgewerteten Datensätze bzw. Krankenhäuser im Jahr 2018 \\
\hline Datenbasis 2018 & Soll & Ist [1] & Vollzähligkeit (\%) \\
\hline Krankenhäuser & 1090 & 1085 & 99,3 \\
\hline 09/1 Implantationen & 917 & 916 & 99,9 \\
\hline 09/2 Aggregatwechsel & 897 & 891 & 99,2 \\
\hline 09/3 Revisionen/Systemwechsel/Explantationen \\
\hline Eingriffe \\
\hline 09/1 Implantationen & 75.466 & 75.522 & 100,1 \\
\hline 09/2 Aggregatwechsel & 15.851 & 16.068 & 101,4 \\
\hline 09/3 Revisionen/Systemwechsel/Explantationen & 10.942 & 10.965 & 100,2 \\
\hline $\begin{array}{l}\text { Die Angaben zur Anzahl der meldenden Krankenhäuser und zur Menge der Datensätze in D Tab. } 3 \\
\text { weichen von den Einträgen in D Tab. 1 ab, da auch Minimaldatensätze mitgezählt werden }\end{array}$ \\
\hline
\end{tabular}

Berichts noch nicht bekannt ist, wer dem neuen Expertengremium angehören wird.

Grundlage des Berichts sind wie jedes Jahr die Bundesauswertungen der für die jeweiligen Verfahren der externen Qualitätssicherung publizierten Daten [1], für einige Vergleiche wurden zudem die Daten vorausgegangener Berichte des Deut- schen Herzschrittmacher- und Defibrillator-Registers verwendet [2].

Die im Folgenden vorgestellten und kommentierten Ergebnisse der Operationen bei Patienten mit Herzschrittmachern (Teil 1) und implantierbaren KardioverterDefibrillatoren (ICD; Teil 2) aus dem stationären Bereich im Jahr 2019 in Deutschland geben aufgrund der immer weniger werdenden Daten, die für die Qualitätssicherung erhoben werden, einen deutlich gröberen Überblick über die Situation bei kardialen Rhythmusimplantaten als zuvor. Der traditionelle Vergleich mit den Registern aus Schweden und der Schweiz ist dennoch möglich [3,4]. Das dänische Herzschrittmacher- und Defibrillator-Register hat für das Jahr 2019 ebenfalls wieder einen Bericht vorgelegt [5].

Die Zahlen aus dem ambulanten Bereich fehlen wie immer, so dass dieser Bericht nicht über alle in Deutschland durchgeführten Eingriffe bei Herzschrittmachern berichtet. Es entzieht sich unserer Kenntnis, wie viele Operationen ambulant durchgeführt werden, und ein vergleichbares Register für ambulante Eingriffe bei Patienten mit Herzschrittmacher ist unseres Wissens nicht vorhanden.

\section{Datenbasis}

\section{Datenvolumen}

Die Anzahl der Institutionen, die Herzschrittmacheroperationen durchführen, geht seit 2011 kontinuierlich zurück. Die Zahl der Neuimplantationen nimmt wie die Zahlen für die Aggregatwechsel erstmals seit Jahren nicht mehr ab, nur die Revisionen sind weiter zurückgegangen. Insgesamt ist die Summe der Schrittmacheroperationen in $2019 \mathrm{im}$ Vergleich zum Vorjahr nahezu gleichgeblieben (• Tab. 1).

Die Zahl der Institutionen, die nur sehr gelegentlich Schrittmacheroperationen durchführen, d.h. weniger als 20 Eingriffe pro Jahr, ist im Jahr 2019 bei allen Eingriffsarten zurückgegangen (• Tab. 2).

Bei den Institutionen mit 20 und mehr Eingriffen pro Jahr gab es keine nennenswerten Veränderungen der Zahlen.

Die Datenvollständigkeit ist weiter nahezu perfekt (• Tab. 3 und 4). Bei den Zahlen über $100 \%$ handelt es sich am ehesten um Kodierfehler oder Doppelerfassungen.

\section{Demografische Daten}

Die Zahl der Eingriffe pro Institution hat bei den Implantationen und Aggregatwechseln geringfügig zugenommen und bei den Revisionen abgenommen. Die Verweildauer ist bei allen Eingriffsarten zurückgegangen. Die Rate an permanent 
Tab. 4 Vollzähligkeit der ausgewerteten Datensätze bzw. Krankenhäuser im Jahr 2019

\begin{tabular}{|c|c|c|c|}
\hline Datenbasis 2019 & Soll & Ist [1] & Vollzähligkeit (\%) \\
\hline \multicolumn{4}{|l|}{ Krankenhäuser } \\
\hline 09/1 Implantationen & 1076 & 1073 & 99,7 \\
\hline 09/2 Aggregatwechsel & 905 & 907 & 100,2 \\
\hline 09/3 Revisionen/Systemwechsel/Explantationen & 878 & 879 & 100,1 \\
\hline \multicolumn{4}{|l|}{ Eingriffe } \\
\hline 09/1 Implantationen & 75.762 & 75.760 & 100,0 \\
\hline 09/2 Aggregatwechsel & 16.191 & 16.338 & 100,9 \\
\hline 09/3 Revisionen/Systemwechsel/Explantationen & 10.533 & 10.523 & 99,9 \\
\hline
\end{tabular}

Tab. 5 Demografische Daten zu Implantationen, Aggregatwechsel und Revisionen/ Systemwechsel/Explantationen

\begin{tabular}{|c|c|c|c|c|}
\hline & & 2017 & 2018 & 2019 \\
\hline \multirow{7}{*}{$\begin{array}{l}09 / 1 \\
\text { Implan- } \\
\text { tationen }\end{array}$} & Anzahl Eingriffe & 77.283 & 75.437 & 75.666 \\
\hline & - Im Mittel je Institution & 70,1 & 69,5 & 70,5 \\
\hline & Männliche Patienten & $56,7 \%$ & $56,9 \%$ & $57,4 \%$ \\
\hline & Weibliche Patienten & $43,3 \%$ & $43,1 \%$ & $42,7 \%$ \\
\hline & Patienten $<60$ Jahre & $6,2 \%$ & $6,0 \%$ & $5,7 \%$ \\
\hline & Patienten 90 Jahre und älter & $5,4 \%$ & $5,5 \%$ & $5,6 \%$ \\
\hline & Mittlere postoperative Verweildauer (Tage) & 4,6 & 4,5 & 4,3 \\
\hline \multirow{8}{*}{$\begin{array}{l}09 / 2 \\
\text { Aggregat- } \\
\text { wechsel }\end{array}$} & Anzahl Eingriffe & 17.085 & 16.061 & 16.333 \\
\hline & - Im Mittel je Institution & 17,9 & 17,5 & 18,0 \\
\hline & Männliche Patienten & $52,7 \%$ & $52,8 \%$ & $54,3 \%$ \\
\hline & Weibliche Patienten & $47,3 \%$ & $47,2 \%$ & $45,8 \%$ \\
\hline & Patienten $<60$ Jahre & $5,2 \%$ & $5,0 \%$ & $4,9 \%$ \\
\hline & Patienten 90 Jahre und älter & $13,3 \%$ & $14,0 \%$ & $15,1 \%$ \\
\hline & Mittlere postoperative Verweildauer (Tage) & 1,8 & 1,7 & 1,6 \\
\hline & Kein Eigenrhythmus & $20,3 \%$ & $21,6 \%$ & $23,1 \%$ \\
\hline \multirow{7}{*}{$\begin{array}{l}\text { 09/3 } \\
\text { Revisionen, } \\
\text { System- } \\
\text { wechsel, } \\
\text { Explan- } \\
\text { tationen }\end{array}$} & Anzahl Eingriffe & 11.383 & 10.933 & 10.498 \\
\hline & - Im Mittel je Institution & 12,3 & 12,3 & 11,9 \\
\hline & Männliche Patienten & $55,0 \%$ & $55,1 \%$ & $56,6 \%$ \\
\hline & Weibliche Patienten & $45,0 \%$ & $44,9 \%$ & $43,4 \%$ \\
\hline & Patienten $<60$ Jahre & $10,8 \%$ & $10,3 \%$ & $10,0 \%$ \\
\hline & Patienten 90 Jahre und älter & $6,2 \%$ & $5,6 \%$ & $5,9 \%$ \\
\hline & Mittlere postoperative Verweildauer (Tage) & 4,9 & 5,0 & 4,7 \\
\hline
\end{tabular}

Tab. 6 Führende EKG-Indikationen zur Schrittmacherimplantation in Absolutzahlen sowie in Prozent im Vergleich zum Vorjahr

\begin{tabular}{|l|l|l|l|l|}
\multirow{2}{*}{ EKG Befunde } & \multicolumn{2}{l}{$\mathbf{2 0 1 8}$} & $\mathbf{2 0 1 9}$ \\
\cline { 2 - 5 } & $n$ & $\%$ & $n$ & $\%$ \\
\hline AV-Block & 35.588 & 47,2 & 36.404 & 48,1 \\
\hline Sick-Sinus-Syndrom (SSS) ${ }^{\mathrm{a}}$ & 25.781 & 34,2 & 25.201 & 33,3 \\
\hline Vorhofflimmern & 10.036 & 13,3 & 9812 & 13,0 \\
\hline Schenkelblöcke & 893 & 1,2 & 954 & 1,3 \\
\hline Indikation zu CRT & 2030 & 2,7 & 2365 & 3,1 \\
\hline Sonstiges & 492 & 0,7 & 595 & 0,8 \\
\hline Fehlende Angaben & 790 & 1,0 & 335 & 0,4 \\
\hline Summe & 75.610 & 100 & 75.666 & 100 \\
\hline anklusive Bradykardie-Tachykardie-Syndrom (BT bei paroxysmalem/persistierendem Vorhofflimmern)
\end{tabular}

stimulationsbedürftigen, d.h. schrittmacherabhängigen Patienten wird nur noch bei den Aggregatwechseln ermittelt und nimmt dort über die Jahre betrachtet kontinuierlich zu. Demgegenüber hat der Anteil an jungen Patienten ( $<60$ Jahre) in allen Eingriffsklassen abgenommen, wohingegen Eingriffe bei betagten Patienten (>90 Jahre) in allen Bereichen wieder zugenommen haben. Bei den übrigen Ergebnissen zeigen sich erwartungsgemäß keine auffälligen Veränderungen (• Tab. 5).

\section{Implantationen}

\section{EKG-Indikation zur Schrittmacher- implantation}

Bei den Indikationen zur Schrittmacherimplantation haben im Jahr 2019 die Indikationsgruppen AV-Block, Schenkelblöcke, die Indikationen zur CRT sowie die Restegruppe "Sonstiges" in Absolutzahlen zugenommen, die Indikationsgruppen Sick-Sinus-Syndrom und bradykardes Vorhofflimmern haben wie die fehlenden Angaben abgenommen. Bei der relativen Verteilung ergeben sich die gleichen Verschiebungen (- Tab. 6).

Die Leitlinienkonformität ist im Jahr 2019 mit 93,55\% im Vergleich zu 2018 mit $92,77 \%$ leicht angestiegen. Bezogen auf die einzelnen Indikationen liegt die Leitlinienkonformität für die AV-Blockierungen $\geq 2$. Grades bei $100 \%$ und für die Bradykardie mit permanentem Vorhofflimmern bei über $97 \%$, für die Sinusknotenerkrankung bei knapp über $90 \%$ und für alle anderen Rhythmusstörungen unter $90 \%$ [1]. Als mögliche Erklärung für die geringere Leitlinienkonformität bei den unter $90 \%$ liegenden Ergebnissen ist, neben dem vergleichsweisen selteneren Auftreten dieser Rhythmusstörungen, die bisweilen herausfordernde Umsetzung von Leitlinientexten in Parameter für die externe Qualitätssicherung zu nennen. Die unterschiedliche Interpretation der Leitlinientexte mag auch der Grund für die weiterhin enttäuschend niedrige Leitlinienkonformität bei der Indikation zur kardialen Resynchronisationstherapie (CRT) sein $(64,57 \%$ in 2019 vs. $59,36 \%$ in 2018 ) sein. 

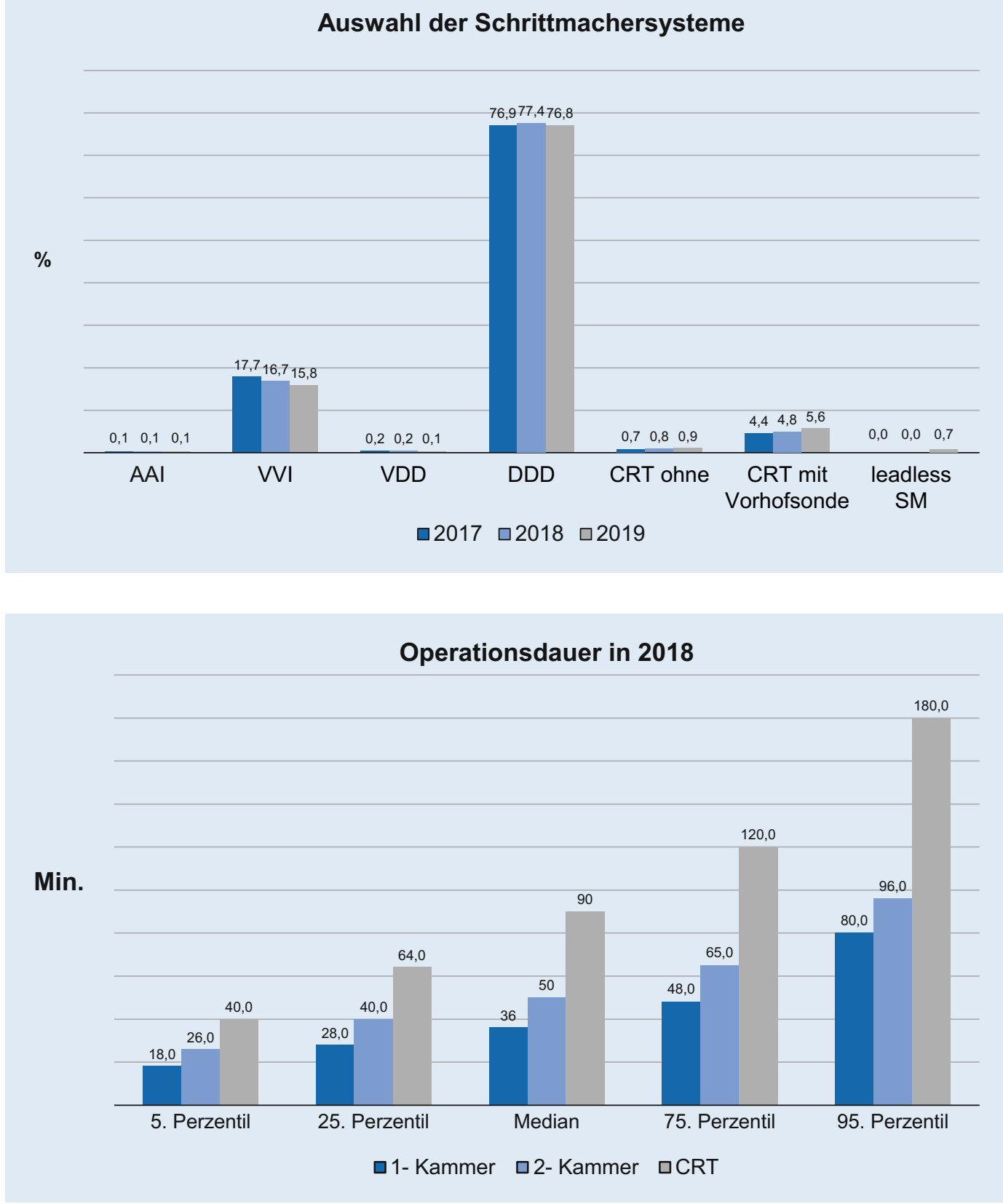

Abb. $1 \varangle$ Prozentuale Verteilung der Schrittmachersysteme bei Implantation 2019 im Vergleich zu den Vorjahren
Abb. $2 \triangleleft$ Perzentile der Operationszeiten im Jahr 2018. (Beispiel: Im Jahr 2018 waren $5 \%$ aller 1-Kammer-Implantationen nach 18 min beendet)

\section{Auswahl der Schrittmachersysteme}

Die Auswahl der Schrittmachersysteme zeigt weiterhin, dass in der täglichen Praxis überwiegend VVI-Systeme oder DDD-Systeme implantiert werden ( $\bullet$ Abb. 1). Dabei hat die Zahl der VVI-Systeme weiter abgenommen und die Zahl der CRT-Systeme mit Vorhofsonde erneut zugenommen.

Die Leitlinienkonformität bei der Systemauswahl liegt unverändert bei über $98 \%$ [1].

Die Hersteller der verwendeten Aggregate werden seit 2017 ebenso wie die
Polarität und die Fixationsmechanismen der verwendeten Sonden nicht mehr erfasst. Dies wird sich auch so schnell nicht ändern, da das inzwischen immerhin gesetzlich verankerte und in Anfängen auch vorhandene Implantateregister Deutschland (IRD) zwar seit dem 26. Mai 2020 in die Zuständigkeit des Bundesministeriums für Gesundheit (BMG) übergegangen ist, aber eine Zunahme der Dynamik bei der praktischen Umsetzung nicht erkennbar ist. Mit einer Aufnahme des Regelbetriebs zumindest für 1 Medizinprodukt, die Brustimplantate, wird inzwischen nicht mehr vor 2023 gerechnet. Zudem wurde vom BMG die initial geplante Aufnahme des Deutschen Herzschrittmacherund Defibrillator-Registers in das übergeordnete deutsche Register verschoben.

\section{Operationsdaten}

Bis 2016 zeigten die Ergebnisse beim venösen Zugang einen kontinuierlichen Trend in die falsche Richtung im Sinne einer ständigen Abnahme der relativen Häufigkeit der Verwendung der V. cephalica für den venösen Zugang. Bis 2019 stieg 
Tab. 7 Venöser Zugang bei Schrittmacherimplantationen $2019 \mathrm{im}$ Vergleich zu den Vorjahren

\begin{tabular}{|l|l|l|l|l|l|l|}
\hline \multirow{2}{*}{ Venöser Zugang } & \multicolumn{2}{|l|}{2017} & \multicolumn{2}{l|}{2018} & 2019 & \\
\cline { 2 - 7 } & $n$ & $\%$ & $n$ & $\%$ & $n$ & $\%$ \\
\hline Vena cephalica & 30.796 & 39,9 & 30.317 & 40,2 & 30.444 & 40,2 \\
\hline Vena subclavia & 49.232 & 63,7 & 47.484 & 63,0 & 46.648 & 61,7 \\
\hline Andere & 1697 & 2,2 & 2085 & 2,8 & 2969 & 3,9 \\
\hline
\end{tabular}

Tab. 8 Operationszeiten in Minuten bei Implantationen 2018 und 2019 (bezogen auf alle Fälle mit gültiger Angabe zur Operationsdauer $>0$ )

\begin{tabular}{|l|l|l|l|}
\hline \multirow{2}{*}{ SM-System } & $\mathbf{2 0 1 8}$ & $\mathbf{2 0 1 9}$ \\
\cline { 2 - 4 } & Median & $n$ & Median \\
\hline 1-Kammer & 36 & 11.966 & 37 \\
\hline 2-Kammer & 50 & 57.961 & 50 \\
\hline CRT & 90 & 4878 & 88 \\
\hline Leadless SM & N/A & 516 & 43 \\
\hline Gesamt & 50 & 75.666 & 50 \\
\hline
\end{tabular}

Tab. 10 Ergebnisse der Reizschwellenmessungen und Bestimmungen der intrakardialen Signalamplituden bei Implantationen (jeweils bezogen auf alle Fälle mit gültiger Angabe)

\begin{tabular}{|c|c|c|c|c|c|}
\hline \multicolumn{2}{|c|}{ Sonde/Messung } & \multirow{2}{*}{$\begin{array}{l}2018 \\
\text { Median }\end{array}$} & \multicolumn{3}{|l|}{2019} \\
\hline & & & $n$ & MW & Median \\
\hline \multirow[t]{2}{*}{ Vorhofsonde } & Reizschwelle (V) & 0,7 & 55.080 & 0,8 & 0,8 \\
\hline & P-Wellen-Amplitude (mV) & 2,8 & 60.620 & 3,1 & 2,8 \\
\hline \multirow[t]{2}{*}{ Ventrikel } & Reizschwelle (V) & 0,6 & 75.315 & 0,7 & 0,6 \\
\hline & R-Wellen-Amplitude (mV) & 11,6 & 72.624 & 12,1 & 11,2 \\
\hline LV-Sonde & Reizschwelle (V) & 1,0 & 4277 & 1,1 & 1,0 \\
\hline
\end{tabular}

Tab. 11 Perioperative Komplikationen bei Implantationen in den Jahren 2016, 2017 und 2019 im Vergleich

\begin{tabular}{|c|c|c|c|c|c|c|}
\hline \multirow[t]{2}{*}{ Perioperative Komplikationen } & \multicolumn{2}{|l|}{2017} & \multicolumn{2}{|l|}{2018} & \multicolumn{2}{|l|}{2019} \\
\hline & $n$ & $\%$ & $n$ & $\%$ & $n$ & $\%$ \\
\hline Asystolie & 105 & 0,1 & \multirow{2}{*}{\multicolumn{2}{|c|}{ k.A. }} & \multirow{2}{*}{\multicolumn{2}{|c|}{ k.A. }} \\
\hline Kammerflimmern & 24 & 0,0 & & & & \\
\hline Kardiopulmonale Reanimation & \multicolumn{2}{|l|}{ k. A. } & 96 & 0,1 & 96 & 0,1 \\
\hline Pneumothorax & 347 & 0,5 & 374 & 0,5 & 352 & 0,5 \\
\hline Perikarderguss & 160 & 0,2 & 167 & 0,2 & 170 & 0,2 \\
\hline Taschenhämatom & 94 & 0,1 & 68 & 0,1 & 88 & 0,1 \\
\hline Hämatothorax & 43 & 0,1 & 35 & 0,1 & 28 & 0,0 \\
\hline Wundinfektion (CDC) & 13 & 0,0 & 16 & 0,0 & 17 & 0,0 \\
\hline Sondendislokation & 922 & 1,2 & 899 & 1,2 & 1019 & 1,4 \\
\hline Sondendysfunktion & 209 & 0,3 & 203 & 0,3 & 253 & 0,3 \\
\hline Sonst. interventionspflichtige Komplikation & 84 & 0,1 & 99 & 0,1 & 68 & 0,1 \\
\hline Mindestens eine perioperative Komplikation & 1871 & 2,4 & 1868 & 2,5 & 1992 & 2,6 \\
\hline
\end{tabular}

Tab. 9 Dosisflächenprodukt 2019 im Vergleich zu den Vorjahren Dosisflächenprodukt (cGy * $\left.\mathrm{cm}^{2}\right)$

\begin{tabular}{|l|l|l|l|}
\hline & $\mathbf{2 0 1 7}$ & $\mathbf{2 0 1 8}$ & $\mathbf{2 0 1 9}$ \\
\hline MW & 887 & 778 & 722 \\
\hline Median & 395 & 356 & 328 \\
\hline 75. Perzentil & 893 & 805 & 752 \\
\hline
\end{tabular}

dann die Verwendung der V. cephalica an und ist 2019 zumindest gleichgeblieben (- Tab. 7). Die Zahl der Institutionen, in denen die Präparation der V. cephalica überhaupt nicht gelingt, wurde auch 2019 (noch) nicht ausgewertet. Wir wissen aber immerhin, dass im Vergleich zu 2018 die Zahl der Patienten, bei denen ausschließlich die V. subclavia als Zugangsweg verwendet wurde, im Jahr 2019 sowohl absolut von 43.176 auf 42.453 als auch relativ von $57,23 \%$ auf $56,49 \%$ abgenommen hat. Dass der Zugangsweg über die V. subclavia durch eine höhere Rate an methodenimmanenten Komplikationen belastet ist und damit die Patientensicherheit kompromittiert, wird seit Jahren an dieser Stelle erwähnt, und scheint als Botschaft seit 2017 angekommen zu sein. Zu der Entscheidung, den venösen Zugangsweg als Qualitätsindikator zu verwenden, wie dies z.B. in Hessen der Fall ist, hat sich das IQTIG bislang für die Bundesauswertung nicht durchringen können, obwohl die Evidenz für die Vorteile des Zugangsweges über die V. cephalica auch außerhalb dieses Registers Jahr für Jahr zunimmt [6].

Die Operationszeiten sind in 0 Tab. 8 dargestellt; sie sind im Vergleich zum Vorjahr nahezu unverändert geblieben. Erstmals sind auch die Ergebnisse für die sog. sondenlosen Schrittmacher verfügbar. Es zeigt sich, dass deren Implantationszeiten zwischen denen für ein konventionelles 1-Kammer-bzw. 2-Kammer-System liegen.

Die Konstanz der Ergebnisse wird bestätigt, wenn man die Ergebnisse für die einzelnen Perzentilen der Operationszeiten aus dem Jahr 2018 (『 Abb. 2) mit den Resultaten aus dem Jahr 2019 (• Abb. 3) vergleicht. In $\square$ Abb. 3 werden erstmals die Ergebnisse für die sondenlosen Schrittmacher ("leadless" SM) angegeben, die die vorherigen Aussagen zur Operationsdauer bestätigen.

Die Auswertungen des Dosisflächenprodukts sind (•Tab.9) zu entnehmen. Die Ergebnisse weisen darauf hin, dass die Strahlenbelastung für Patienten und Operateure erfreulicherweise weiter abnimmt.

An der Konstanz der Ergebnisse für die Reizschwellenbestimmung sowie für die Ermittlung der intrakardialen Signalamplituden hat sich auch 2019 so gut wie nichts geändert (• Tab. 10). Seit 2017 werden die 

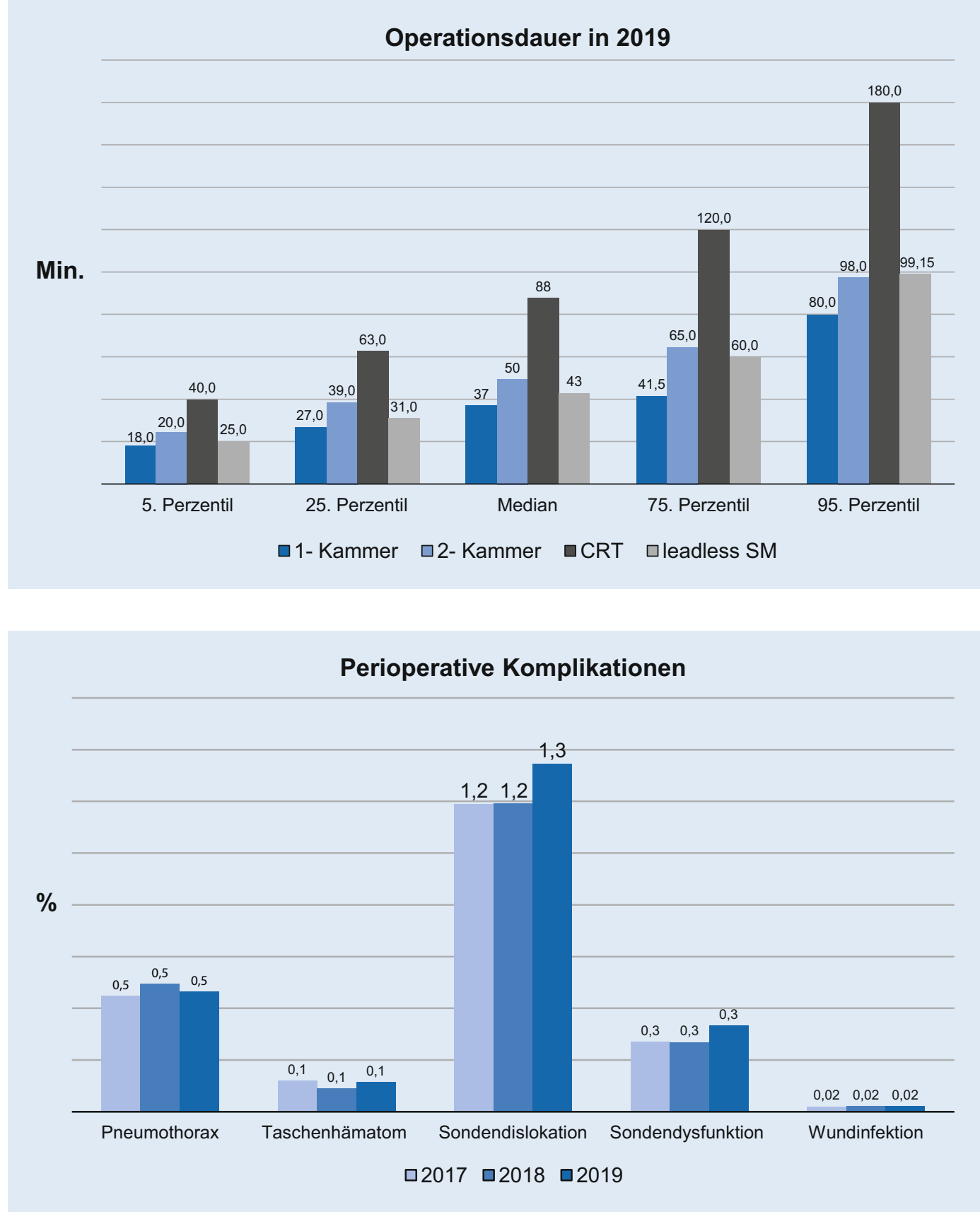

Abb. $3<$ Perzentile der Operationszeiten im Jahr 2019. (Beispiel: Im Jahr 2019 waren $5 \%$ aller 1-Kammer-Implantationen nach $18 \mathrm{~min}$ beendet)
Abb. $4 \triangleleft$ Überblick über die perioperativen Komplikationen nach Implantation
Ergebnisse der Amplitudenhöhe des linksventrikulären Signals nicht mehr erhoben.

Die Ergebnisse bei den perioperativen Komplikationen zeigen weiter nur wenig Veränderungen und haben insgesamt insbesondere bei den Sondenkomplikationen erneut leicht zugenommen (- Abb. 4 und Tab. 11). Die Komplikationsarten Asystolie und Kammerflimmern sind seit 2018 weggefallen. Dafür ist die Komplikationsart kardiopulmonale Reanimation hinzugekommen, wobei die kardiopulmonale Reanimation genau genommen keine Komplikation, sondern den Versuch der Verhinderung bzw. der Behandlung einer solchen darstellt.

Für die Sondendislokationen und die Sondendysfunktionen sind die Ergebnisse detaillierter in 0 Tab. 12 dargestellt. Es zeigt sich sowohl bei den Dislokationen als auch bei den Dysfunktionen eine Zunahme bei den Vorhof- und Ventrikelsonden im Vergleich zum Vorjahr; die Ergebnisse für die LV-Sonde sind in einem zahlenmäßigen Bereich, der einen Vergleich nicht sinnvoll erscheinen lässt.
Auf die an dieser Stelle üblicherweise dargestellten Ergebnisse der Sonderauswertungen für den Zusammenhang zwischen der Gesamtkomplikationsrate und dem venösen Zugangsweg sowie zwischen Fallzahlen und Komplikationen müssen wir bedauerlicherweise auch für das Jahr 2019 noch verzichten.

\section{Aggregatwechsel}

Die Zahl dieser Eingriffe ist im Jahr 2019 im Gegensatz zu den Vorjahren zum ersten 
Tab. 12 Perioperative Komplikationen bei Sonden in den Jahren 2016, 2017 und 2019 (jeweils bezogen auf alle Fälle mit dem jeweiligen Sondenproblem, z. B. im Jahr 2016 ereigneten sich 484 bzw. 57, $1 \%$ aller Sondendislokationen im Vorhof)

\begin{tabular}{|l|l|l|l|l|l|l|}
\hline \multirow{2}{*}{ Sondenprobleme } & 2017 & 2018 & 2019 \\
\cline { 2 - 8 } & $n$ & $\%$ & $n$ & $\%$ & $n$ & $\%$ \\
\hline Dislokationen & 551 & 59,8 & 575 & 64,0 & 685 & 67,2 \\
\hline Vorhofsonde & 388 & 42,1 & 348 & 38,7 & 359 & 35,2 \\
\hline 1. Ventrikelsonde & 14 & 1,5 & 9 & 1,0 & 13 & 1,3 \\
\hline LV-Sonde & 0 & 0,0 & 0 & 0,0 & $\leq 3$ & 0,1 \\
\hline Beide Ventrikelsonden & \multicolumn{7}{|l|}{} & \multicolumn{3}{l|}{} \\
\hline Dysfunktionen & 56 & 26,8 & 45 & 22,2 & 86 & 34,0 \\
\hline Vorhofsonde & 160 & 76,6 & 164 & 80,8 & 186 & 73,5 \\
\hline 1. Ventrikelsonde & 6 & 2,9 & 5 & 2,5 & 0 & 0,0 \\
\hline LV-Sonde & 0 & 0,0 & 0 & 0,0 & 0 & 0,0 \\
\hline Beide Ventrikelsonden &
\end{tabular}

\begin{tabular}{|c|c|c|c|c|c|c|}
\hline \multirow[t]{2}{*}{ Art der Komplikation } & \multicolumn{2}{|c|}{2017} & \multicolumn{2}{|c|}{2018} & \multicolumn{2}{|c|}{2019} \\
\hline & $n$ & $\%$ & $n$ & $\%$ & $n$ & $\%$ \\
\hline Asystolie & 7 & 0,04 & \multirow{2}{*}{\multicolumn{2}{|c|}{ k. A. }} & \multirow{2}{*}{\multicolumn{2}{|c|}{ k. A. }} \\
\hline Kammerflimmern & 0 & 0,00 & & & & \\
\hline Kardiopulmonale Reanimation & \multicolumn{2}{|c|}{ k. A. } & 5 & 0,03 & $\leq 3$ & 0,01 \\
\hline Taschenhämatom & 16 & 0,09 & 10 & 0,08 & 13 & 0,08 \\
\hline Wundinfektion (CDC) & $\leq 3$ & 0,01 & $\leq 3$ & 0,02 & 4 & 0,02 \\
\hline Sonstige interventionspflichtige Komplikation & 10 & 0,06 & 11 & 0,07 & 8 & 0,05 \\
\hline Mindestens eine perioperative Komplikation & 34 & 0,20 & 27 & 0,17 & 27 & 0,17 \\
\hline
\end{tabular}

\begin{tabular}{|l|l|l|l|l|l|l|}
\hline Tab. 14 Ort des letzten Eingriffs, welcher der Revisionsoperation vorausging \\
\hline $\begin{array}{l}\text { Ort des letzten Eingriffs vor der } \\
\text { aktuellen Operation }\end{array}$ & $\mathbf{2 0 1 7}$ & $\mathbf{2 0 1 8}$ & $\mathbf{2 0 1 9}$ & \\
\cline { 2 - 8 } & $n$ & $\%$ & $n$ & $\%$ & $n$ & $\%$ \\
\hline Eigene Institution, stationär & 7083 & 62,2 & 6875 & 62,9 & 6661 & 63,5 \\
\hline Eigene Institution, ambulant & 79 & 0,7 & 59 & 0,5 & 69 & 0,7 \\
\hline Andere Institution, stationär & 4099 & 36,0 & 3903 & 35,7 & 3661 & 34,9 \\
\hline Andere Institution, ambulant & 122 & 1,1 & 96 & 0,9 & 107 & 1,0 \\
\hline Alle Eingriffe & 11.383 & 100 & 10.933 & 100 & 10.498 & 100 \\
\hline
\end{tabular}

Tab. 15 Indikation zur Revisionsoperation. (Mehrfachnennung möglich, ab 2016 zudem 2 neue, zusätzliche Schrittmacher-Aggregat-Probleme: vorzeitiger Aggregataustausch anlässlich einer Revisionsoperation/eines Systemwechsels und sonstige aggregatbezogene Indikation)

\begin{tabular}{|l|l|l|l|l|l|l|}
\hline \multirow{2}{*}{ Indikation zur Revision } & $\mathbf{2 0 1 7}$ & $\mathbf{2 0 1 8}$ & $\mathbf{2 0 1 9}$ \\
\cline { 2 - 7 } & $n$ & $\%$ & $n$ & $\%$ & $n$ & $\%$ \\
\hline Schrittmacher-Aggregat-Problem & 5040 & 36,1 & 4930 & 33,9 & 4675 & 33,6 \\
\hline Taschenproblem & 1645 & 11,8 & 1784 & 12,3 & 1677 & 12,0 \\
\hline Sondenproblem & 7280 & 52,1 & 7837 & 53,9 & 7578 & 54,4 \\
\hline
\end{tabular}

Mal seit 2015 wieder angestiegen. Demgegenüber hat die Zahl der Krankenhäuser, die diese Art von Eingriff durchführen, weiter abgenommen (• Tab. 1).

Die Detailgenauigkeit der Auswertungen hat auch bei den Aggregatwechseln in einer aus Sicht des Registers bedauerlichen Art und Weise nachgelassen. So werden inzwischen weder die Laufzeiten noch die jeweiligen Unterschiede je nach Schrittmachersystem oder -hersteller ausgewertet. Damit wird ein aus Sicht von Patienten und Ärzten wichtiger Qualitätsaspekt der Therapie mit kardialen Rhythmusimplantaten nicht mehr beleuchtet.

Die absolute und relative Häufigkeit von Komplikationen nach Austauschoperationen ist nahezu gleichgeblieben ( $\bullet$ Tab. 13). Die für das Jahr 2019 eingeführten Änderungen bei der Datenerfassung wurden bereits bei den Betrachtungen zu $\bullet$ Tab. 11 kommentiert.

\section{Revisionen/Systemwechsel/ Explantationen}

Die Zahl der Krankenhäuser, die diese Eingriffe, die im Folgenden als Revisionen zusammengefasst werden, durchführen, hat im Jahr 2019 ebenso wie die Zahl der Revisionen im Vergleich zu den Vorjahren weiter abgenommen (• Tab. 1).

Die Zahl der Revisionen bei Patienten, die zuvor am eigenen Hause operiert wurden, hat an relativer Häufigkeit zugenommen (• Tab. 14).

Bei den Indikationen zur Revision haben die Zahlen bei allen Komplikationsarten abgenommen, die Rate an Sondenproblemen hat dabei leicht zugenommen (• Tab. 15).

Bei der Detailbetrachtung der Schrittmacher-Aggregatprobleme zeigt sich erneut eine unerwartete Zunahme der Fehlfunktionen/Rückrufe sowie der Zahl der Aggregatperforationen (ब Tab. 16). Insbesondere Ersteres bedarf der weiteren Beobachtung. Alle anderen Aggregatprobleme 
Tab. 16 Indikation zur Revisionsoperation bei Schrittmacher-Aggregat- oder Taschenproblemen bzw. Systemumwandlungen. (Mehrfachnennungen möglich)

\begin{tabular}{|l|l|l|l|l|l|l|}
\hline \multirow{2}{*}{ Schrittmacher-Aggregat-Problem } & $\mathbf{2 0 1 7}$ & $\mathbf{2 0 1 8}$ & $\mathbf{2 0 1 9}$ \\
\cline { 2 - 7 } & $n$ & $\%$ & $n$ & $\%$ & $n$ & $\%$ \\
\hline Batterieerschöpfung & 2925 & 43,8 & 2915 & 43,4 & 2814 & 44,3 \\
\hline Fehlfunktion/Rückruf & 189 & 2,8 & 156 & 2,3 & 235 & 3,7 \\
\hline Aggregataustausch bei Revision & 1063 & 15,9 & 1021 & 15,2 & 880 & 13,9 \\
\hline Anderes Aggregatproblem & 863 & 12,9 & 838 & 12,5 & 746 & 11,7 \\
\hline Taschenhämatom & 91 & 1,4 & 101 & 1,5 & 105 & 1,7 \\
\hline Infektion & 716 & 10,7 & 862 & 12,8 & 787 & 12,4 \\
\hline Aggregatperforation & 212 & 3,2 & 212 & 3,2 & 253 & 4,0 \\
\hline Anderes Taschenproblem & 626 & 9,4 & 609 & 9,1 & 532 & 8,4 \\
\hline Summe & 6685 & 100 & 6714 & 100 & 6352 & 100 \\
\hline
\end{tabular}

Tab. 17 Indikationen zur Revisionsoperation bei Sondenproblemen. (Eine Anzahl von $\leq 3$ wird nicht mehr gesondert ausgewiesen)

\begin{tabular}{|l|l|l|l|}
\hline \multirow{2}{*}{ Sondenprobleme 2019 } & \multicolumn{3}{l}{ Sonde } \\
\cline { 2 - 4 } & Vorhof & Ventrikel & Ventrikel \\
\cline { 3 - 4 } & & RV & LV \\
\hline Dislokation & 1712 & 794 & 80 \\
\hline Sondenbruch/Isolationsdefekt & 411 & 759 & 20 \\
\hline Fehlerhafte Konnektion & 56 & 57 & $\leq 3$ \\
\hline Zwerchfell/Pektoraliszucken & 17 & 79 & 22 \\
\hline Inhibition durch Muskelpotentiale/Oversensing & 80 & 121 & 4 \\
\hline Wahrnehmungsfehler/Undersensing & 102 & 236 & $\leq 3$ \\
\hline Stimulationsverlust/Reizschwellenanstieg & 434 & 1852 & 72 \\
\hline Infektion & 672 & 760 & 77 \\
\hline Myokardperforation & 79 & 228 & 4 \\
\hline Sonstige & 216 & 244 & 46 \\
\hline Summe & 3779 & 5130 & 329 \\
\hline
\end{tabular}

Tab. 18 Relative Häufigkeit der Indikationen zur Revisionsoperation bei Sondenproblemen. (Prozentzahlen bezogen auf die Summe der Probleme der jeweiligen Sonde)

\begin{tabular}{|l|l|l|l|}
\hline \multirow{2}{*}{ Sondenprobleme 2019} & Sonde & \multicolumn{3}{l|}{} \\
\cline { 2 - 4 } & \multirow{2}{*}{$\begin{array}{l}\text { Vorhof } \\
\text { (\%) }\end{array}$} & Ventrikel & Ventrikel \\
\cline { 3 - 4 } & RV (\%) & LV (\%) \\
\hline Dislokation & 45,3 & 15,5 & 24,3 \\
\hline Sondenbruch/Isolationsdefekt & 10,9 & 14,8 & 6,1 \\
\hline Fehlerhafte Konnektion & 1,5 & 1,1 & 0,6 \\
\hline Zwerchfell/Pektoraliszucken & 0,4 & 1,5 & 6,7 \\
\hline Inhibition durch Muskelpotentiale/Oversensing & 2,1 & 2,4 & 1,2 \\
\hline Wahrnehmungsfehler/Undersensing & 2,7 & 4,6 & 0,6 \\
\hline Stimulationsverlust/Reizschwellenanstieg & 11,5 & 36,1 & 21,9 \\
\hline Infektion & 17,8 & 14,8 & 23,4 \\
\hline Myokardperforation & 2,1 & 4,4 & 1,2 \\
\hline Sonstige & 5,7 & 4,8 & 14,0 \\
\hline
\end{tabular}

sind gleichgeblieben oder zurückgegangen.

Bei den Sondenproblemen im Vorhof bleibt die Dislokation mit Abstand die häufigste Komplikation und hat erneut deutlich an Zahl zugenommen, wohingegen bei der rechtsventrikulären Sonde Reizschwellenprobleme die häufigste Komplikation darstellen. In zahlenmäßig relevanter, nahezu gleicher Häufigkeit stellten zudem Dislokationen, Sondenbrüche oder Isolationsdefekte sowie Infektionen die Indikationen zur Revision dar (- Tab. 17 und 18).

Bei den insgesamt seltenen Problemen der LV-Sonde sind Dislokation, Infektionen und Reizschwellenprobleme die häufigsten Ursachen für einen Revisionseingriff. Wie es bei in der Regel fehlenden Wahrnehmungsfunktionen für die LV-Sonde dennoch zu Wahrnehmungsproblemen kommen kann, bleibt ebenso unbeantwortet wie dieFrage, ob insbesondere die Verwendung epikardialer LV-Sonden häufiger zu Myokardperforationen geführt hat, oder ob die Verwendung dieser Sonden keinen Einfluss auf diese Komplikationsart hatte.

Daten zum bis 2018 publizierten Zeitabstand zwischen Revisionsoperation und dem vorausgegangenen Eingriff werden seit 2019 nicht mehr angegeben.

Bei der chirurgischen Korrektur von Sondenproblemen (- Abb. 5) wird weiterhin sowohl im Vorhof aber insbesondere im Ventrikel eine Neuimplantation bevorzugt.

Daten für das Vorgehen bei der Explantation funktionsloser Sonden, das wir bislang in einer eigenen Abbildung dargestellt haben, werden seit 2018 bei Revisionen von Herzschrittmachersonden nicht mehr erhoben.

Die Komplikationen nach Revisionsoperationen haben im Gegensatz zum Vorjahr an absoluter und relativer Häufigkeit wieder abgenommen (- Tab. 19). Die erneut unglaublich niedrige Rate an Infektionen nach Revisionen ist vermutlich zum größten Teil der kurzen stationären Ver- 
Tab. 19 Perioperative Komplikationen bei Revision, Systemumstellung, Explantation

\begin{tabular}{|c|c|c|c|c|c|c|}
\hline \multirow[t]{2}{*}{ Art der Komplikation } & \multicolumn{2}{|c|}{2017} & \multicolumn{2}{|c|}{2018} & \multicolumn{2}{|c|}{2019} \\
\hline & $n$ & $\%$ & $n$ & $\%$ & $n$ & $\%$ \\
\hline Asystolie & 11 & 0,10 & \multirow{2}{*}{\multicolumn{2}{|c|}{ k. A. }} & \multirow{2}{*}{\multicolumn{2}{|c|}{ k. A. }} \\
\hline Kammerflimmern & 7 & 0,06 & & & & \\
\hline Kardiopulmonale Reanimation & \multicolumn{2}{|l|}{ k. A. } & 17 & 0,16 & 15 & 0,14 \\
\hline Pneumothorax & 34 & 0,30 & 22 & 0,20 & 29 & 0,28 \\
\hline Hämatothorax & 12 & 0,11 & 9 & 0,08 & 8 & 0,08 \\
\hline Perikarderguss & 16 & 0,14 & 24 & 0,22 & 27 & 0,26 \\
\hline Taschenhämatom & 15 & 0,13 & 17 & 0,16 & 17 & 0,16 \\
\hline Sondendislokation & 54 & 0,47 & 51 & 0,69 & 49 & 0,47 \\
\hline Sondendysfunktion & 16 & 0,14 & 30 & 0,40 & 22 & 0,21 \\
\hline Wundinfektion & 6 & 0,05 & $\leq 3$ & 0,03 & 8 & 0,08 \\
\hline Sonst. Komplikation & 18 & 0,16 & 27 & 0,25 & 14 & 0,13 \\
\hline Mindestens eine perioperative Komplikation & 176 & 1,55 & 203 & 1,86 & 169 & 1,61 \\
\hline
\end{tabular}

Tab. 20 Sterblichkeit im Krankenhaus bei Implantationen, Aggregatwechseln und Revisionen/ Systemwechseln/Explantationen $2019 \mathrm{im}$ Vergleich zu den Ergebnissen in 2016 und 2017

\begin{tabular}{|l|l|l|l|l|l|l|l|}
\hline \multirow{2}{*}{ Tod bei oder nach } & \multicolumn{2}{l|}{2017} & \multicolumn{2}{l|}{2018} & \multicolumn{2}{l|}{2019} \\
\cline { 2 - 8 } & $n$ & $\%$ & $n$ & $\%$ & $n$ & $\%$ \\
\hline Erstimplantation & 1144 & 1,44 & 1040 & 1,38 & 1007 & 1,33 \\
\hline Aggregatwechsel & 42 & 0,25 & 28 & 0,17 & 28 & 0,17 \\
\hline Revision, Systemwechsel oder Explantation & 183 & 1,61 & 194 & 1,77 & 167 & 1,59 \\
\hline
\end{tabular}

Tab. 21 Datenbasis im internationalen Vergleich

\begin{tabular}{|c|c|c|c|c|}
\hline & Dänemark $^{a}$ & Schweiz $^{b}$ & Schwedenc & Deutschland $^{d}$ \\
\hline Meldende Institutionen & 14 & 74 & 43 & 1073 \\
\hline Implantierende Institutionen & 14 & 75 & 43 & 1073 \\
\hline Erstimplantationen & 4498 & 5781 & 7476 & 75.760 \\
\hline - Im Mittel je Institution & 534 & 77 & 174 & 71 \\
\hline - Pro 1 Mio. Einwohner & 772 & 672 & 724 & 911 \\
\hline Folgeeingriffe & 1192 & 1678 & 3202 & 26.861 \\
\hline $\begin{array}{l}\text { Verhältnis Erstimplantation/ } \\
\text { Folgeeingriffe }\end{array}$ & 6,27 & 3,45 & 2,33 & 2,82 \\
\hline Summe & 5690 & 7459 & 10.678 & 102.621 \\
\hline \multicolumn{5}{|c|}{$\begin{array}{l}\text { 'Einwohner in Dänemark am 31.12.2019: 5.827.460 (Quelle: [9]) } \\
\text { bEinwohner in der Schweiz am 31.12.2019: 8.606.033 (Quelle: [10]) } \\
\text { 'Einwohner in Schweden am 31.12.2019: 10.327.590 (Quelle: [11]) } \\
\text { dEinwohner in Deutschland am 31.12.2019: 83.166.700 (Quelle: [12]) }\end{array}$} \\
\hline
\end{tabular}

Tab. 22 Prozentuale Verteilung venöser Zugänge bei Neuimplantationen im Vergleich

\begin{tabular}{|l|l|l|l|}
\hline & Schweiz (\%) & Schweden (\%) & Deutschland (\%) \\
\hline V. cephalica & 32,9 & 47,6 & 38,0 \\
\hline V. subclavia & 55,0 & 51,5 & 58,3 \\
\hline Andere & 12,1 & 0,9 & 3,7 \\
\hline
\end{tabular}

weildauer geschuldet, die eine Detektion der Infektion innerhalb eines stationären Aufenthalts verhindert. Leider werden Infektionen nach Revisionsoperationen auch nicht durch den vor 2 Jahren neu eingeführten Qualitätsindikator 2195 „Infektionen oder Aggregatperforationen als Indikation zum Folgeeingriff innerhalb eines Jahres" erfasst, da dieser Indikator nur die Neuimplantationen und bestimmte Arten von Systemumwandlungen erfasst.

Die Sterblichkeit ist weiter nach Neuimplantationen und Revisionen am höchsten, wobei die Ursache bei den Neuimplantationen nach wie vor nicht vollständig erklärbar ist; bei einem gewissen Anteil dieser Gruppe dürfte es sich um multimorbide Patienten handeln, die akut höhergradige Bradykardien entwickeln und konsekutiv einen Herzschrittmacher erhalten, aber im weiteren Verlauf an ihrer Grunderkrankung versterben. Demgegenüber ist die Beobachtung, dass die Sterblichkeit nach Revisionen höher ist als nach Austauschoperationen verständlich. Allerdings ist bei allen Ergebnissen zur Sterblichkeit zu bedenken, dass nicht der Tod aufgrund der Prozedur, sondern die Sterblichkeit während des stationären Aufenthalts ermittelt wird.

Im Vergleich zu den Vorjahren haben sich die Ergebnisse kaum verändert, die zuvor beobachtete, kontinuierliche Zunahme der Sterblichkeit nach Revisionen war in 2019 nicht mehr vorhanden (• Tab. 20).

\section{Kommentar mit internationalem Vergleich}

\section{Datenbasis}

Traditionsgemäß werden an dieser Stelle die Daten aus Deutschland mit anderen europäischen Registerberichten verglichen. Für das Jahr 2019 liegen wiederum Berichte aus der Schweiz und Schweden vor 

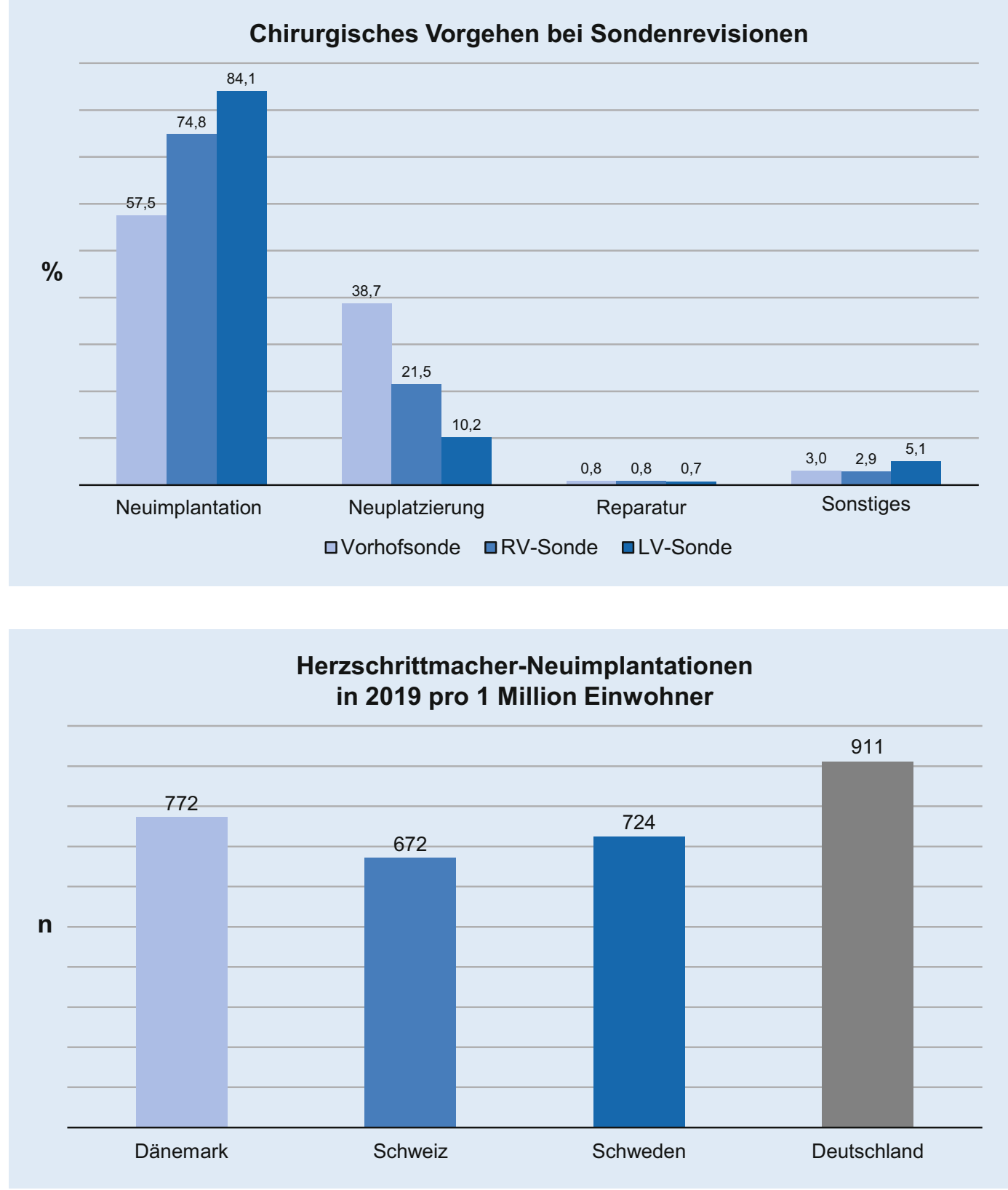

Abb. $5 \triangleleft$ Chirurgisches Vorgehen bei der Sondenrevision. (Bezug: alle postoperativ funktionell aktiven Sonden, an denen ein Eingriff vorgenommen wurde)
Abb. $6<$ Implantationen pro 1 Mio. Einwohner im internationalen Vergleich
$[3,4]$, zudem haben auch die Dänen wieder einen Bericht publiziert [5].

Die Berichte der European Heart Rhythm Association (EHRA; [7]) sowie der britischen Kollegen [8] sind bislang seit der Publikation der Daten von 2017 nicht mehr fortgeführt worden. Überdies waren die Daten wegen ihrer Unvollständigkeit sowie der unterschiedlichen Datenaufbereitung für einen Vergleich nicht geeignet.

Der Vergleich der Datenbasis zeigt das bekannte Bild: In Deutschland werden sowohl absolut (-Tab. 21) als auch relativ die meisten Schrittmacher implantiert
(- Abb. 6). DieZahl der Neuimplantationen pro Einrichtung ist insbesondere in Dänemark, aber auch in Schweden deutlich höher als in der Schweiz oder in Deutschland. In allen Ländern steigen die Implantationsraten nur noch in geringem Ausmaß an.

Die hohe Implantationsrate in Deutschland lässt sich seit Jahren mit den zur Verfügung stehenden Daten nicht plausibel erklären. Das Alter der Patienten bietet bei weitestgehend vergleichbarer Altersstruktur weiterhin keinen Anhalt. Im Vergleich zum Vorjahr ist der Anteil der Hochbetagten in allen Ländern bis auf Schweden angestiegen (• Abb. 7).
EKG-Indikationen zur Schrittmachertherapie

Bei den EKG-Indikationen (• Abb. 8) gibt es kaum Veränderungen: Der AV-Block ist weiterhin in allen 4 Ländern die häufigste Bradykardieform.

\section{Schrittmachersystemauswahl}

Nachdem AAI-Systeme auch in den skandinavischen Ländern kaum noch implantiert werden und auch bei VDD-Systemen mit Ausnahme der Schweiz (1,2\%) keine nennenswerten Implantationsraten mehr 

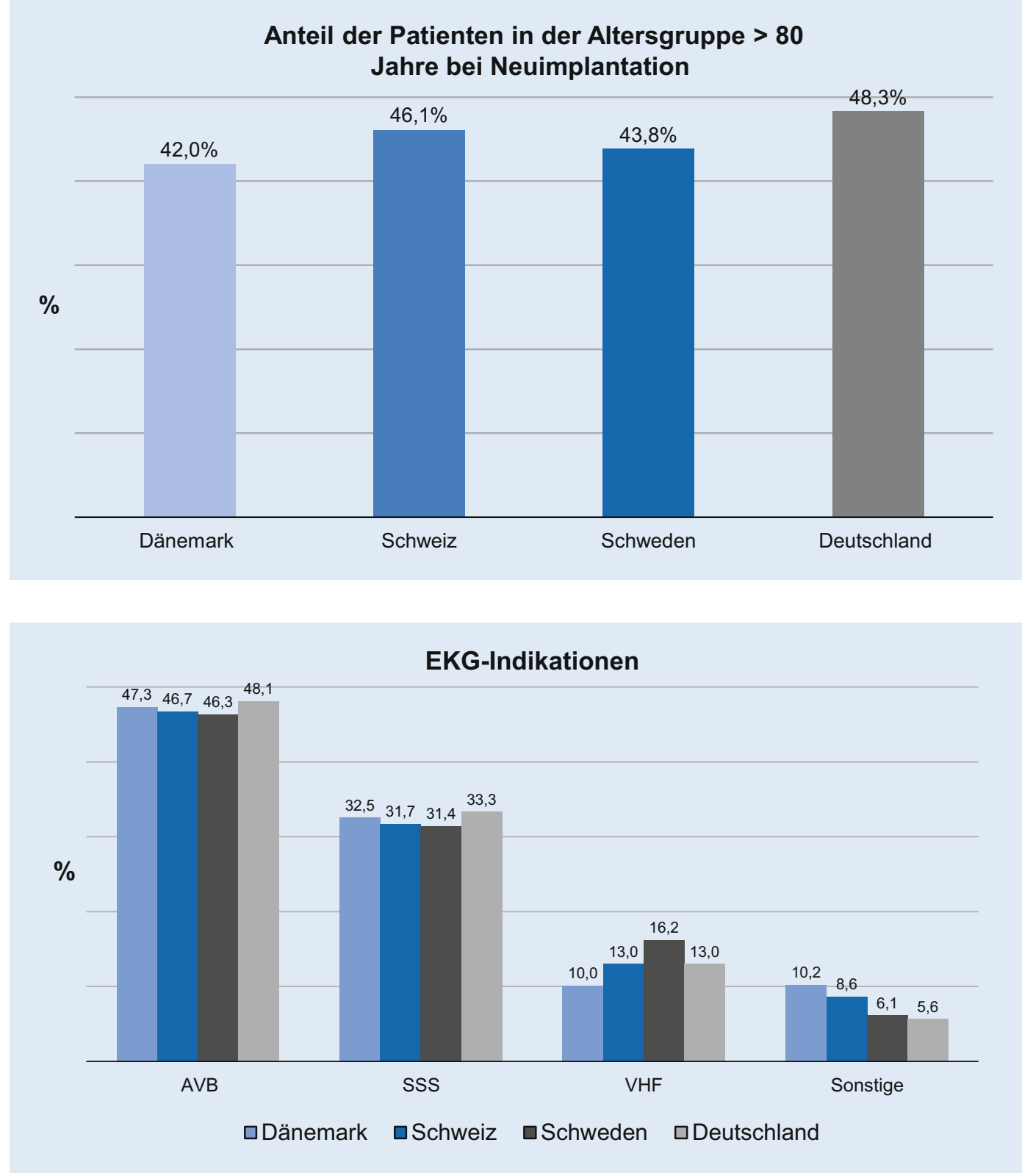

Abb. $7 \triangleleft$ Anteil älterer Patienten $\geq 80$ Jahre (Schweiz $\geq 81$ Jahre) an allen Patienten, bei denen ein Herzschrittmacher in $2019 \mathrm{im}$ plantiert wurde, im Vergleich

\begin{abstract}
Abb. $8<$ EKG-Indikationen im internationalen Vergleich. AVB AV-Block, SSS Sick-Sinus-Syndrom inkl. BTS, VHF bradykardes Vorhofflimmern, Sonstige Sonstige Rhythmusstörungen
\end{abstract}

berichtet werden, sind in 0 Abb. 9 nur die Implantationsraten an VVI-, DDD-, CRTund sondenlosen Systemen aufgeführt. VVI-Systeme werden in Dänemark und in der Schweiz am häufigsten implantiert, DDD-Systeme in Schweden und Deutschland am häufigsten verwendet, wohingegen die Rate der Implantationen von CRTSystemen in Dänemark vergleichsweise am niedrigsten von allen 4 Ländern ist. Sondenlose Schrittmachersysteme wurden in 2019 v. a. in der Schweiz, aber auch in Dänemark in nennenswerter Stückzahl implantiert.

\section{Operationsdaten}

Bei der Verwendung der V. cephalica zum Sondenvorschub gibt es im Jahr 2019 eine deutliche Zunahme der relativen Häufigkeit ihrer Verwendung in der Schweiz und einen Rückgang in Schweden zu verzeichnen, in Deutschland hat sie kaum noch zugenommen. Die Schweden verwenden weiterhin die V. cephalica deutlich häufiger als die Deutschen und diese wiederum häufiger als die Schweizer (• Tab. 22). Die zahlenmäßigen Unterschiede der Daten in - Tab. 22 im Vergleich zu den Angaben in - Tab. 7 sind durch die rechnerische Korrektur der Ergebnisse auf $100 \%$ be- dingt, die zur besseren Vergleichbarkeit der Ergebnisse notwendig wurde. Die Dänen stellen die Verwendung der V. cephalica zusammen für alle Rhythmusimplantate (Herzschrittmacher und ICD) als Ratio von V. cephalica zu V. subclavia dar. Demnach wurde im Jahr 2019 die V. cephalica 2,43mal und damit mehr als doppelt so häufig für den Sondenvorschub verwendet als die V. subclavia.

Bei den Operationszeiten bleiben die Schweden weiterhin schneller als die Deutschen und diese wiederum schneller als die Eidgenossen. Im Vergleich zum Vorjahrfällt auf, dass insbesondere in der Schweiz und in Deutschland die Mittelwerte der Ope- 

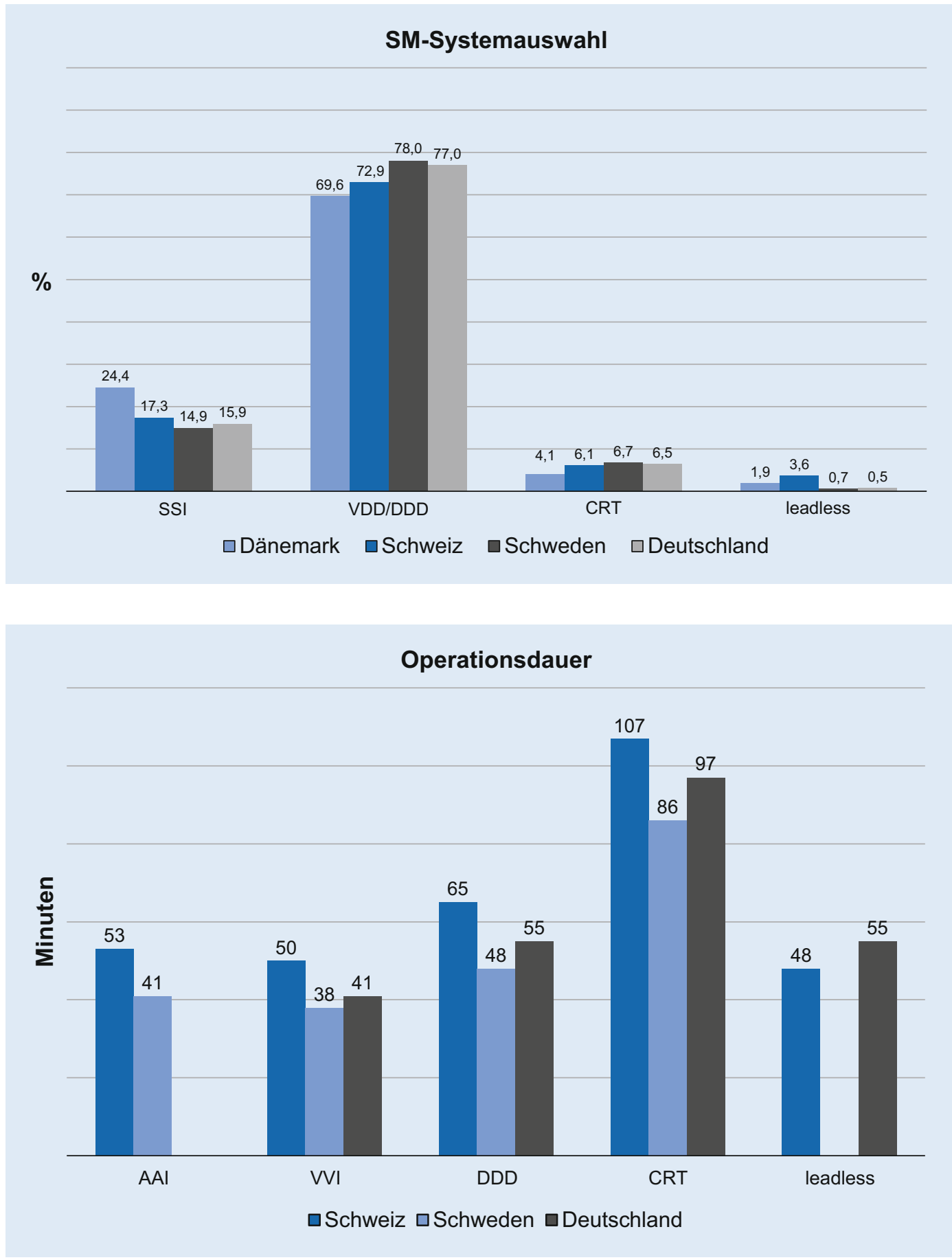

Abb. $9 \triangleleft$ Auswahl des Schrittmachersystems im Vergleich. (SSI 1-KammerSystem [AAl oder VVI], leadless sondenloses Schrittmachersystem)
Abb. $10 \triangleleft$ Vergleich der mittleren Operationsdauer für verschiedene Systeme. (Für Deutschland wurden alle 1-und 2-KammerSysteme als VVIbzw. DDD zusammengefasst, in der Schweiz werden VVI- und VDD-Systeme zusammengefasst) rationsdauern zugenommen haben. Die Unterschiede in den Ergebnissen zwischen - Abb. 10 und 0 Tab. 8 bzw. A Abb. 3 sind dadurch bedingt, dass in - Abb. 10 die Mittelwerte, in $\square$ Tab. 8 bzw. $\triangle$ Abb. 3 die Mediane angegeben sind. Die Mittelwerte wurden verwendet, um eine Vergleichbarkeit der Ergebnisse zwischen den Ländern zu ermöglichen. Die deutsche Auswertung unterteilt zudem bei den 1-Kammer-Sys- temen nicht mehr nach AAI und VVI, so dass die entsprechenden Zahlen nur eingeschränkt vergleichbar sind (- Abb. 10).

Die Auswahl der Vorhof- und Ventrikelsonden zeigte über die Jahre hinweg ein nahezu unverändertes Bild bzw. einen unveränderten Trend: Es werden inzwischen fast ausschließlich bipolare Sonden verwendet, die im Vorhof fast immer und im Ventrikel über die Jahre zunehmend über aktive Fixationsmechanismen verfügten. Da diese Daten in Deutschland seit 2017 nicht mehr erfasst werden, ist kein Vergleich mit anderen Ländern mehr möglich.

\section{Fazit und Ausblick}

Seit Jahren wurden die Registerberichte traditionell abgeschlossen durch die Be- 


\section{Infobox}

\section{Tabellenverzeichnis}

Tab. 1: Übersicht ausgewerteter Datensätze im Vergleich zu den Vorjahren, inkl. Minimaldatensätze Tab. 2: Operationsvolumina (Implantationen, Aggregatwechsel und Revisionen) der meldenden Krankenhäuser

Tab. 3: Vollzähligkeit der ausgewerteten Datensätze bzw. Krankenhäuser im Jahr 2018

Tab. 4: Vollzähligkeit der ausgewerteten Datensätze bzw. Krankenhäuser im Jahr 2019

Tab. 5: Demografische Daten zu Implantationen, Aggregatwechsel und Revisionen/Systemwechsel/ Explantationen

Tab. 6: Führende EKG-Indikationen zur Schrittmacherimplantation in Absolutzahlen sowie in Prozent im Vergleich zum Vorjahr

Tab. 7: Venöser Zugang bei Schrittmacherimplantationen $2019 \mathrm{im}$ Vergleich zu den Vorjahren Tab. 8: Operationszeiten in Minuten bei Implantationen 2018 und 2019 (bezogen auf alle Fälle mit gültiger Angabe zur Operationsdauer $>0$ )

Tab. 9: Dosisflächenprodukt 2019 im Vergleich zu den Vorjahren

Tab. 10: Ergebnisse der Reizschwellenmessungen und Bestimmungen der intrakardialen

Signalamplituden bei Implantationen (jeweils bezogen auf alle Fälle mit gültiger Angabe)

Tab. 11: Perioperative Komplikationen bei Implantationen in den Jahren 2017, 2018 und $2019 \mathrm{im}$ Vergleich

Tab. 12: Perioperative Komplikationen bei Sonden 2017, 2018 und 2019 (jeweils bezogen auf alle Fälle mit dem jeweiligen Sondenproblem, z. B. im Jahr 2017 ereigneten sich 546 bzw. 55,9\% aller Sondendislokationen im Vorhof)

Tab. 13: Perioperative Komplikationen bei Aggregatwechseln

Tab. 14: Ort des letzten Eingriffs, welcher der Revisionsoperation vorausging

Tab. 15: Indikation zur Revisionsoperation. (Mehrfachnennung möglich, ab 2017 zudem 2 neue, zusätzliche Schrittmacher-Aggregat-Probleme: vorzeitiger Aggregataustausch anlässlich einer Revisionsoperation/eines Systemwechsels und sonstige aggregatbezogene Indikation)

Tab. 16: Indikation zur Revisionsoperation bei Schrittmacher-Aggregat- oder Taschenproblemen bzw. Systemumwandlungen. (Mehrfachnennungen möglich)

Tab. 17: Indikationen zur Revisionsoperation bei Sondenproblemen. (Eine Anzahl von <3 wird nicht mehr gesondert ausgewiesen)

Tab. 18: Relative Häufigkeit der Indikationen zur Revisionsoperation bei Sondenproblemen.

(Prozentzahlen bezogen auf die Summe der Probleme der jeweiligen Sonde)

Tab. 19: Perioperative Komplikationen bei Revision, Systemumstellung, Explantation

Tab. 20: Sterblichkeit im Krankenhaus bei Implantationen, Aggregatwechseln und Revisionen/ Systemwechseln/Explantationen 2019 im Vergleich zu den Ergebnissen in 2017 und 2018

Tab. 21: Datenbasis im internationalen Vergleich

Tab. 22: Prozentuale Verteilung venöser Zugänge bei Neuimplantationen im Vergleich

trachtung von drei Ergebnissen, die man als Indikatoren für unterschiedliche Aspekte der Therapie mit Herzschrittmachern bewerten konnte. Die Ergebnisse waren jeweils Resultate von Sonderauswertungen durch die BQS, das AQUA-Institut oder das IQTIG. Momentan bemühen wir uns, diese Daten im Rahmen der sekundären Datennutzung zu erhalten (siehe auch die Einleitung). Aus den in der Einleitung bereits erwähnten Gründen müssen wir die Ergebnisse der Sonderauswertungen aber weiterhin schuldig bleiben, hoffen aber, dass sie in Zukunft zumindest in abgewandelter Form wieder zur Verfügung stehen werden.

Die Zusammenfassung dieses Registerberichts Herzschrittmacher schließt bei den anderen Aspekten erneut nahtlos an die Berichte der vergangenen Jahre an:

1. Die Implantationszahlen in Deutschland zählen weltweit mit zu den höchsten, nehmen aber inzwischen kaum noch zu.

2. Indikation und Systemauswahl erfolgen mit hoher Leitlinienkonformität.

3. Ob Institutionen mit hohem Operationsvolumen schneller und mit weniger Komplikationen operieren, wissen wir auch in 2019 leider nicht; es wird aber wohl so geblieben sein, und

4. die Rate an Revisionsoperationen, insbesondere aufgrund von Sondenproblemen wie der Dislokation oder dem Reizschwellenanstieg, bleibt hoch, nimmt aber nach einem Anstieg im letzten Jahr in 2019 wieder etwas $a b$.

Zum Schluss dieses ersten Teils des Berichtes 2019 möchten wir uns wieder bei allen, die bei der Erstellung dieses Berichts mitgeholfen haben, ganz herzlich bedanken. Wir hoffen, dass die Informationen
Infobox 2

Abbildungsverzeichnis

Abb. 1: Prozentuale Verteilung der Schrittmachersysteme bei Implantation 2019 im Vergleich zu den Vorjahren

Abb. 2: Perzentile der Operationszeiten im Jahr 2018. (Beispiel: Im Jahr 2018 waren $5 \%$ aller 1-Kammer-Implantationen nach $18 \mathrm{~min}$ beendet)

Abb. 3: Perzentile der Operationszeiten im Jahr 2019. (Beispiel: Im Jahr 2019 waren 5\% aller 1-Kammer-Implantationen nach $18 \mathrm{~min}$ beendet)

Abb. 4: Überblick über die perioperativen Komplikationen nach Implantation Abb. 5: Chirurgisches Vorgehen bei der Sondenrevision

Abb. 6: Implantationen pro 1 Mio. Einwohner im internationalen Vergleich

Abb. 7: Anteil älterer Patienten $\geq 80$ Jahre (Schweiz >81 Jahre) an allen Patienten, bei denen ein Herzschrittmacher in 2019 implantiert wurde, im Vergleich

Abb. 8: EKG-Indikationen im internationalen Vergleich

Abb. 9: Auswahl des Schrittmachersystems im Vergleich

Abb. 10: Vergleich der mittleren Operationsdauer für verschiedene Systeme. (Für Deutschland wurden alle 1- und 2-KammerSysteme als VVI bzw. DDD zusammengefasst, in der Schweiz werden VVI- und VDD-Systeme zusammengefasst)

des Berichts dem Anwender in der täglichen Praxis und damit auch den Patienten helfen. Schließlich bleibt der Wunsch, dass bei den Punkten mit Verbesserungspotenzial die Lektüre dieses Berichts dabei hilft die Größe des Problems zu reduzieren.

\section{Korrespondenzadresse}

Prof. Dr. A. Markewitz, OTA a.D.

Am Goldberg 24, 56170 Bendorf, Deutschland AndreasMarkewitz@gmx.de

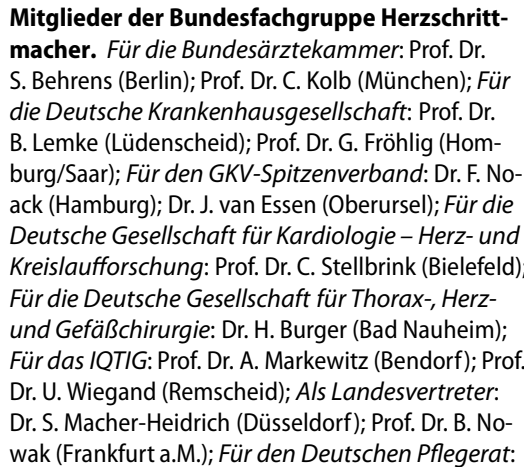




\section{Einhaltung ethischer Richtlinien}

Interessenkonflikt. A. Markewitz gibt an, dass kein Interessenkonflikt besteht.

Für diesen Beitrag wurden von den Autoren keine Studien an Menschen oder Tieren durchgeführt. Für die aufgeführten Studien gelten die jeweils dort angegebenen ethischen Richtlinien.

\section{Literatur}

1. Herzschrittmacherversorgung (HSM). https:// iqtig.org/qs-verfahren/hsm/. Zugegriffen: 4. Mai 2021

2. German Pacemaker Register. https://pacemakerregister.de/.Zugegriffen: 4. Mai 2021

3. Schweizerische Stiftung für Rhythmologie Jahresstatistiken. http://www.rhythmologie-stiftung. ch/statistiken_de.html.Zugegriffen:4. Mai 2021

4. Swedish ICD and Pacemaker Registry Dokumentbank. https://www.pacemakerregistret.se/ icdpmr/docbank.do. Zugegriffen:4. Mai 2021

5. https://www.google.de/search?q=danish+pace maker+and+icd+register+annual+report+2019 \&sxsrf=ALeKk02EmDDC156quNWteATj1XyGL1bF uQ\%3A1620134923569\&source $=h p \& e i=C 0 y R Y N$ 2H9GFjLsP2J-m2AI\&iflsig=AINFCbYAAAAAYJFaG wdXIPipLG1zfwPje6EVrfA-AdH9\&oq=da\&gs_Icp= Cgdnd3Mtd2I6EAEYATIGCCMQJxATMgQ\|xAnMgY IIxAnEBMyBQgAELEDMggIABCxAxCDATIE-

CAAQQzIICAAQsQMQgwwiz. Zugegriffen: 4. Mai 2021

6. Benz AP, Vamos M, Erath JW, Hohnloser SH (2019) Cephalic vs. subclavian leadimplantation in cardiac implantable electronic devices: a systematic review and meta-analysis. Europace 21:121-129

7. https://www.escardio.org/static_file/Escardio/ Subspecialty/EHRA/Publications/Documents/ 2019/ehra-white-book-2019.pdf. Zugegriffen: 2. Okt. 2020

8. NICOR Cardiac rhythm management (Arrhythmia audit). https://www.nicor.org.uk/nationalcardiac-audit-programme/cardiac-rhythmmanagement-arrhythmia-audit/. Zugegriffen: 4. Mai 2021

9. https://www.statbank.dk/statbank5a/selectvarval/ define.asp?PLanguage $=1 \&$ subword $=$ tabsel $\&$ MainTable=FOLK. Zugegriffen: 22. Apr. 2021

10. BFS Bevölkerungsdaten im Zeitvergleich, 1950-2019. https://www.bfs.admin.ch/bfs/ de/home/statistiken/bevoelkerung.assetdetail. 14367961.html.Zugegriffen:22. Apr. 2021

11. SCB Population and population changes 1749-2020. http://www.scb.se/en/findingstatistics/statistics-by-subject-area/population/ population-composition/population-statistics/ pong/tables-and-graphs/yearly-statistics--thewhole-country/population-and-populationchanges/.Zugegriffen:22. Apr. 2021

12. Destatis/Stat. BundesamtBevölkerung in Deutschland im Jahr 2019 auf 83,2 Millionen gestiegen. https://www.destatis.de/DE/Presse/ Pressemitteilungen/2020/06/PD20_223_12411. html.Zugegriffen: 22. Apr. 2021 